\title{
Molecular Tuning of Styryl Dyes Leads to Versatile and Efficient Plasma Membrane Probes for Cell and Tissue Imaging
}

\author{
Mayeul Collot, ${ }^{* 1}$ Emmanuel Boutant, ${ }^{1}$ Kyong Tkhe Fam, ${ }^{1}$ Lydia Danglot, ${ }^{2,3}$ Andrey S. Klymchenko ${ }^{* 1}$ \\ ${ }^{1}$ Laboratoire de Bioimagerie et Pathologies, UMR 7021 CNRS, University of Strasbourg, France. \\ ${ }^{2}$ University Paris Diderot, Sorbonne Paris Cité, Institut Jacques Monod, CNRS UMR 7592, 75013 Paris, France. \\ ${ }^{3}$ INSERM U894, Institute of Psychiatry and Neuroscience of Paris, Membrane Traffic in Healthy and Diseased Brain, 102 \\ rue de la Santé, 75014 Paris, France.
}

Keywords: Fluorescent probes, Plasma membrane probes, bioimaging, neuron, brain slice, plant imaging

\begin{abstract}
The plasma membrane (PM) plays a major role in many biological processes; therefore its proper fluorescence staining is required in bioimaging. Among the commercially available PM probes, styryl dye FM1-43 is one of the most widely used. In this work, we demonstrated that fine chemical modifications of FM1-43 can dramatically improve the PM staining. The newly developed probes, SP-468 and SQ-535 were found to display enhanced photophysical properties (reduced crosstalk, higher brightness, improved photostability) and unlike FM1-43, provided excellent and immediate PM staining in 5 different mammalian cell lines including neurons (primary culture and tissue imaging). Additionally, we showed that the new probes displayed differences in their internalization pathways compared to their parent FM1-43. Finally, we demonstrated that the modifications made to FM1-43 did not impair the ability of the new probes to stain the PM of plant cells. Overall, this work presents new useful probes for PM imaging in cells and tissues and provides insights on the molecular design of new PM targeting molecules.
\end{abstract}

\section{Introduction}

The plasma membrane (PM), as a barrier between the extracellular and intracellular environments, maintains the integrity of various organisms at the cellular level and plays key roles in biology. ${ }^{1,23}$ Consequently, the proper and selective staining of the PM is important in bioimaging to delimit the cells and to continue deciphering its role in various cellular mechanisms. Nowadays, fluorescence microscopy remains the most widely used bioimaging technique as it is relatively affordable and it rapidly provides important information with good resolution. From these two observations, numerous efforts have been made to develop fluorescent PM probes ${ }^{4,5,6,7}$ including from our group. ${ }^{8-15}$ Among them, FM dyes (named after Fei Mao ${ }^{16}$ ), which are styryl dyes introduced by Betz and colleagues in the early $90 \mathrm{~s}$ ' as synaptic vesicle markers, ${ }^{16}$ rapidly became among the most famous and used commercially available PM probes. ${ }^{17,18}$ These amphiphilic dyes were further developed by the group of Loew to give rise to PM voltage sensitive probes $^{19,}{ }^{20}$ and membrane lipid domains probes. ${ }^{21}$ These probes are composed of an aniline or naphthylamine donor linked to a pyridinium acceptor moiety through a double bond or a polymethine chain, which can tune their color. ${ }^{19,}{ }^{20}$ However, efforts were mainly focused on designing probes bearing polar groups on one end and hydrophobic groups on the other end, which thus imposed vertical orientation of styryl fluorophores in the membrane. In the case of FM1-43 (Figure 1), one of the most commonly used FM probes, it was claimed that the dibutyl chains bore by the aniline moiety interact with the hydrocarbon chains of the lipid bilayer via hydrophobic interactions whereas the triethylammonium cationic moiety bore by the pyridinium interacts with the negative charges of the phospholipids through opposite charge attraction. ${ }^{22}$ Unlike hydrophobic carbocyanines, such as DiI, DiD and DiR, bearing two C-18 hydrocarbon chains, ${ }^{17}$ FM dyes display a lower hydrophobicity and thus do not precipitate in aqueous media. Therefore, FM probes are successfully and widely used for imaging PM or vesicle trafficking in plants. ${ }^{23,}{ }^{24}$ In mammalian cells they were applied to study vesiculation ${ }^{25,26}$ and to image recycled synaptic vesicles in neuroscience. ${ }^{27,28,29}$ Unfortunate- ly, the reduced hydrophobicity of FM1-43 leads to a lack of affinity towards the PM and inefficient PM staining in fixed cells. ${ }^{4}$ Moreover, FM probes tend to internalize quickly by endocytosis ${ }^{30}$ and their broad absorption and emission spectra limit their use in multicolor imaging. Nevertheless, attractive optical properties of FM dyes, such as their photostability, can constitute an advantage for long-term imaging and their large Stokes shifts is important Stimulated Emission Depletion (STED) super resolution imaging. ${ }^{31,32}$ We recently introduced a family of PM probes with balanced hydrophobicity that provided efficient PM staining for cells and tissue, namely MemBright.9, ${ }^{14}$ These PM probes were obtained by decorating BODIPY $^{9}$ and cyanines ${ }^{14}$ with a clickable and selective PM targeting moiety: $\mathrm{CAZ}^{10}$ (Scheme 1, Figure 1).

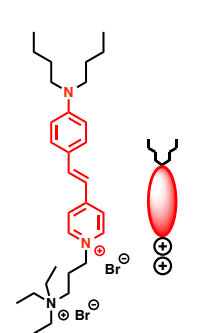

FM-1-43

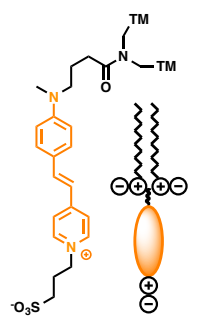

$\mathrm{SP}-468$

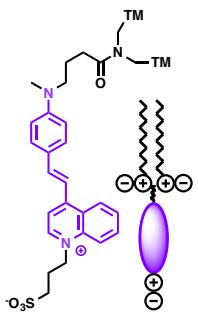

SQ-535
TM= Targeting Moietie III
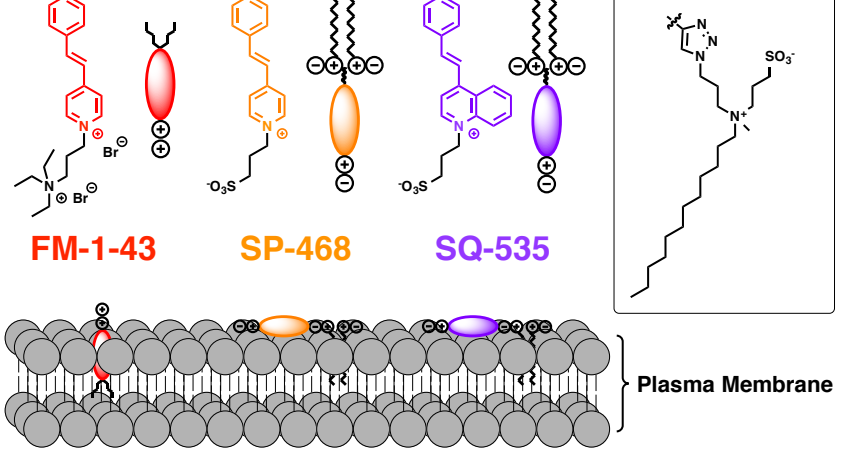

Figure 1. Structures of FM1-43 and the newly developed styryl probes SP-468 (Styryl Pyridinium - $\lambda_{\text {Ex } \max } 468$ ) and SQ-535 (Styryl Quinolinium - $\lambda_{\mathrm{Ex} \max } 535$ ) and their orientation within the plasma membrane.

We herein showed that the staining properties of FM dyes can be dramatically improved by means of fine chemical modifications. By neutralizing the global charge, inserting polar head groups at both ends of the probe, tuning the hydrophobicity and optionally extending the electronic system of the dye, we 
obtained two new optimized version of FM1-43, namely SP468 and SQ-535 (Figure 1) that efficiently and rapidly stain the PM in both eukaryotic and plant cells.

\section{Results and discussion}

\section{Design of the probes}

FM1-43 is a red emitting plasma membrane probe from the FM family. It is a styryl dye composed of an $N, N$-dialkylated aniline donor moiety linked through an ethylene connector to a 4-pyridinium acceptor moiety (Figure 1). It exhibits some affinity to PM due to two synergic interactions. First, the butyl groups bring hydrophobicity to enhance the affinity towards the hydrophobic tails of the lipids, while the cationic pyridinium and the ammonium moieties interact with the polar head of the lipids. ${ }^{22}$ In order to improve the PM imaging properties of FM1-43, we performed three different modifications. 1) We first chose to annihilate the cationic nature of FM dyes since cationic and hydrophobic molecules tend to accumulate inside the cells. ${ }^{41}$ Therefore, the ammonium group of FM was replaced by a sulfonate group to give rise to a neutral zwiterionic dye (Figure 1). 2) Then, one of the two butyl groups borne by the aniline moiety of FM1-43 was replaced by a methyl group in order to reduce the inductive donor effect that electronically enriches the nitrogen. This modification should provoke a hypsochromic shift in the absorption spectrum and thus should reduce the cross talk phenomenon due to the relatively high extinction coefficient of FM1-43 at $530 \mathrm{~nm}$ and $560 \mathrm{~nm}$ that are generally dedicated to excitation sources for the red channel in fluorescence microscopy. 3) Finally, two amphiphilic moieties were introduced by CuAAc click chemistry to ensure an efficient PM targeting. 9 All these modifications led to a probe called SP-468 (Styryl Pyridinium). As an additional modification, the pyridinium moiety of the fluorophore has been replaced by a quinolinium in order to extend the $\pi$ conjugation of the fluorophore. ${ }^{42}$ This extension led to SQ-535 and is expected to provoke a bathochromic shift in both absorbance and emission spectra, which would ensure its use in the red channel with limited crosstalk in the green one.

\section{Synthesis}

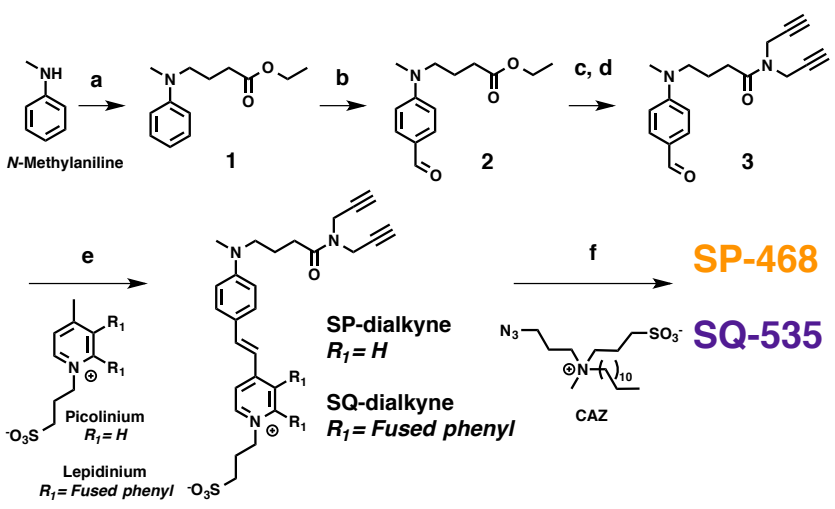

Scheme 1. Synthesis of SP-468 and SQ-535. a) Ethyl 4bromobutyrate, $\mathrm{NaI}, \mathrm{NaHCO}_{3}, \mathrm{DMSO}, 80^{\circ} \mathrm{C}$. b) $\mathrm{POCl}_{3}, \mathrm{DMF}$, $55^{\circ} \mathrm{C}$. c) $\mathrm{NaOH}(1 \mathrm{M}), \mathrm{MeOH}$. d) Dipropargylamine, DIEA, HATU, DMF, RT. e) Picolinium propane-1-sulfonate or lepidinium propane-1-sulfonate, piperidine (cat.), $\mathrm{MeOH}, 110^{\circ} \mathrm{C}$. f) $\mathrm{CAZ}$, $\mathrm{CuSO}_{4} \cdot 5 \mathrm{H}_{2} \mathrm{O}, \mathrm{AscNa}$, water, DMF, $60^{\circ} \mathrm{C}$.
The synthesis started with the $N$-alkylation of $N$-methylaniline to introduce an ester group (Scheme 1). The aniline 1 was then formylated using the Vilsmeier reaction to obtain 2. The ester was saponified and the obtained carboxylic acid was directly coupled to dipropargylamine using HATU as a coupling agent to give compound 3. This aldehyde was then involved in Knoevenagel reaction with picolinium propane-1-sulfonate and lepidinium propane-1-sulfonate to obtain SP-dialkyne and SQ-dialkyne respectively. These styryl dyes were clicked to $\mathrm{CAZ}^{10}$ to finally lead to the probes SP-468 and SQ-535 (Scheme 1).

\section{Spectroscopic studies}

To evaluate their photophysical properties, the absorption and emission spectra of the three styryl dyes were measured in various conditions. Methanol and DMSO were chosen as polar and solubilizing solvents, whereas PBS was chosen as a typical aqueous medium for cell imaging microscopy. In order to mimic the presence of plasma membrane, liposomes composed of DOPC (1,2-Dioleoyl-sn-glycero-3-phosphocholine) were used in the presence of the dyes. Their photophysical properties are reported in table 1 .

Table 1. Photophysical properties of the probes.

\begin{tabular}{|c|c|c|c|c|c|c|c|c|}
\hline Probe & Solvent & $\begin{array}{c}\lambda_{\text {Abs max }} \\
(\mathrm{nm})\end{array}$ & $\begin{array}{c}\lambda_{\text {Em max }} \\
(\mathrm{nm})\end{array}$ & $\begin{array}{l}\text { FWHM } \\
\text { (Abs) }\end{array}$ & $\begin{array}{c}\text { FWHM } \\
(\mathrm{Em})\end{array}$ & $\begin{array}{l}\text { Stockes Shift } \\
(\mathrm{nm})\end{array}$ & QY & $F / F_{0}{ }^{d}$ \\
\hline \multirow{4}{*}{ FM1-43 } & PBS & 497 & 635 & 95 & $N / A^{b}$ & 138 & 0.01 & 1 \\
\hline & $\mathrm{MeOH}$ & 509 & 630 & 84 & $N / A^{b}$ & 121 & 0.02 & 2 \\
\hline & DMSO & 499 & 637 & 86 & $N / A^{b}$ & 138 & 0.05 & 8 \\
\hline & Liposomes $^{\mathrm{a}}$ & 486 & 602 & 90 & 99 & 116 & 0.15 & 31 \\
\hline \multirow{4}{*}{ SP-468 } & PBS & 472 & 613 & 97 & 85 & 141 & 0.03 & 1 \\
\hline & $\mathrm{MeOH}$ & 485 & 615 & 85 & 81 & 130 & 0.03 & 1 \\
\hline & DMSO & 483 & 625 & 86 & 81 & 142 & 0.07 & 2 \\
\hline & Liposomes $^{a}$ & 468 & 600 & 80 & 91 & 132 & 0.26 & 8 \\
\hline \multirow{4}{*}{ SQ-535 } & PBS & 525 & N/A & 114 & $N / A^{c}$ & $N / A^{c}$ & $N / A^{c}$ & 1 \\
\hline & $\mathrm{MeOH}$ & 555 & 664 & 104 & $N / A^{b}$ & 109 & 0.01 & 4 \\
\hline & DMSO & 557 & 704 & 108 & $N / A^{b}$ & 147 & 0.002 & 8 \\
\hline & Liposomes $^{a}$ & 535 & 665 & 103 & 99 & 130 & 0.10 & 53 \\
\hline
\end{tabular}

${ }^{\mathrm{a}}$ Liposomes (size $100 \mathrm{~nm}$ ) are composed of DOPC (200 $\mu \mathrm{M}$ final concentration in phosphate buffer $20 \mathrm{mM}$, pH 7.4).

b The low fluorescence intensity did not allow to determine the FWHM from the spectrum

${ }^{c}$ No proper fluorescence spectrum was obtained.

${ }^{\mathrm{d}}$ Fluorescence enhancement: $\mathrm{F}=$ Fluorescence intensity at $\lambda_{\mathrm{Em} \max }$ in the presence of liposomes, $F_{0}=$ fluorescence intensity at the same wavelength in PBS.

Similarly to the parent probe FM1-43, the new probes showed hypochromism in water compared to organic solvents (Figure $\mathrm{S} 1$ ), and quantum yields in water and methanol were low (Table 1). However, the newly designed SP-468 displayed significant differences compared to FM1-43 (Figure 2A). First, a hypsochromic shift was observed in all solvents, in both absorption (up to $25 \mathrm{~nm}$ ) and emission (up to $22 \mathrm{~nm}$ ). As expected and discussed above, the replacement of a butyl chain for a methyl group decreased the electron density of the nitrogen donor, thus provoking the blue shift. In the presence of liposomes, the new probes showed significant blue shift in the absorption and emission spectra compared to water and polar organic solvents, similarly to that of FM1-43. However, their Stokes shift was much larger, reaching $130 \mathrm{~nm}$. This larger Stokes shift could be explained by a different insertion of the probes, where the fluorophore of new probes will adopt a parallel orientation to the membrane surface. 

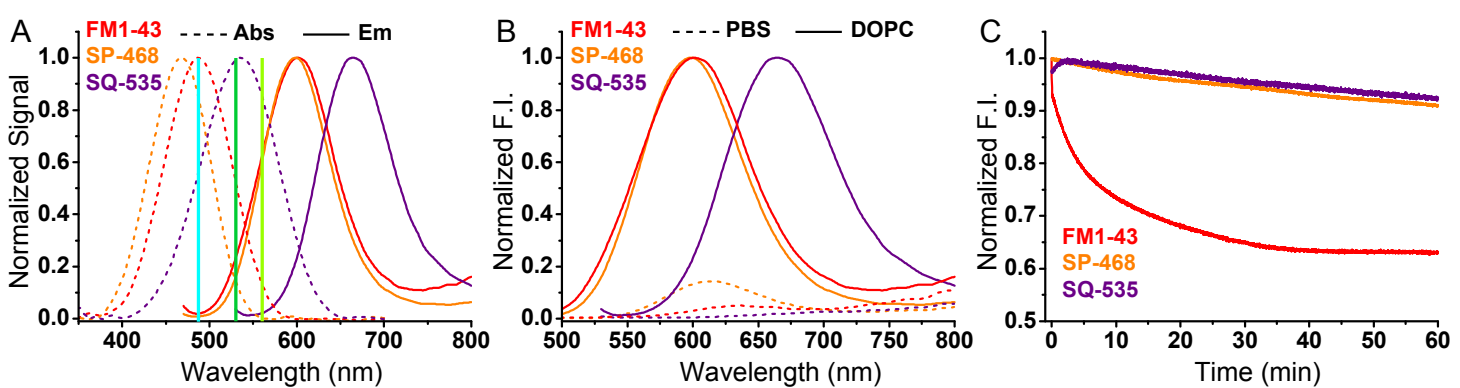

Figure 2. (A) Normalized absorption and emission spectra of the styryl probes in the presence of DOPC vesicles $(200 \mu \mathrm{M})$. The 3 vertical lines indicate the most common laser lines used in imaging namely: 488, 530 and $560 \mathrm{~nm}$. Absorption spectra in presence of liposomes were corrected from scattering. (B) Normalized fluorescence spectra depicting the fluorescence enhancement of the probes from PBS to insertion into DOPC vesicles $(200 \mu \mathrm{M})$. (C) Photostability: evolution of the fluorescence intensity over the time under continuous irradiation. The probes were excited at wavelengths corresponding to the same optical density values (0.03). Concentration of the probes was 2 $\mu \mathrm{M}$

It was noteworthy that SP-468 displayed a sharper absorption band (FWHM= $80 \mathrm{~nm}$ ) compared to FM1-43 (90 nm) (Figure 2A), whereas both SP-468 and FM1-43 are efficiently excited by the commonly used 488-nm laser ( 84 and $99 \%$ respective1y). Advantageously, SP-468 with a blue shifted excitation wavelength combined to a sharper peak is less prone to crosstalk compared to FM1-43. Indeed, while FM1-43 is also well excitable at 530 and $560 \mathrm{~nm}(52 \%$ and $13 \%$ respectively), SP468 is only excited at 14 and $3 \%$ at these laser wavelengths (see laser lines on Figure 2A). The presence of liposomes resulted in significant enhancement of fluorescence intensity for all three probes, which according to the earlier studies indicates that the probe is located in highly organized environment of lipid membranes that minimize rotation-induced quenching in the styryl dyes. However, in case of SP-468, the quantum yield in PBS was slightly higher compared to FM1-43, which decreased fluorescence enhancement from PBS to liposomes ( 8 -fold vs 31 fold, respectively, Figure 2B). The monitoring of the fluorescence signal over the time and in the presence of lipid vesicles indicated that FM1-43 and SP-468 immediately incorporated the lipid bilayer, whereas SQ-535 showed binding within less than a minute (Figure S2). We can conclude that both FM1-43 and SP-468 are present in PBS in molecular form, showing very fast binding kinetics. On the other hand, a more hydrophobic SQ-535 is probably partially aggregated in PBS, which leads to slower binding kinetics, similarly to previously reported MemBright probes. ${ }^{8}$ The aggregation of SQ535 in PBS is in line with the strong blue shift in absorbance compared to organic solvents, which are much less pronounced in case of FM1-43 and SP-468. Extending the $\pi$ system from SP-468 to SQ-535 led to a 65-67 nm red shift in both absorption and emission spectra of dyes in DOPC liposomes (Figure 2A). SQ-535 constitutes a suitable PM probe with high fluorescence enhancement 53-fold, figure 2B). Although it is well excitable at $488 \mathrm{~nm}$ (57\%), SQ-535 starts emitting at only $560 \mathrm{~nm}$ and thus can be used in combination with green emitting dye like BODIPY, fluorescein or eGFP (vide infra). We then assessed the photostability of the probes in the presence of DOPC liposomes and it was noteworthy that FM1-43 photobleached in an exponential manner (30\% decrease of the fluorescence intensity after $15 \mathrm{~min}$ irradiation), while after $1 \mathrm{~h} \mathrm{SP-468}$ and SQ-535 lost only $10 \%$ and $8 \%$ of their fluorescence intensity respectively (Figure 2C). This difference might be assigned to the design of the probes that impose fluorophore of SP-468 and SQ-535 to locate parallel to the membrane plane, in contrast to FM1-43 where the fluorophore itself insert perpendicular to the lipid bilayer. ${ }^{22}$

\section{Cellular imaging}

Prior to imaging studies, the effect of the probes on the cell viability was assessed by MTT assay. As expected, whereas SP-468 and SQ-535 did not show significant signs of cytotoxicity up to $5 \mu \mathrm{M}$, the cationic nature of the FM1-43 provoked a notable decrease of the cell viability after $24 \mathrm{~h}$ at various concentrations (Figure S3). As a first imaging experiment, the ability of FM1-43 and SP-468 to stain the PM of live cells was evaluated on 4 different eukaryotic cell lines at a concentration of $1 \mu \mathrm{M}$ in no-wash conditions. Since FM1-43 and SP-468 possess similar photophysical properties, the same image acquisition and processing settings were applied for both. The results showed that in the same conditions, whereas FM1-43 failed at providing any signal, SP-468 immediately displayed a selective and intense staining of the PM regardless the cell line (Figure 3). When the laser power was increased, the images obtained with FM1-43 were of poor quality with a weak signal/background ratio even after 20 minutes whereas SP-468 maintained a high quality staining over that same period (Figure S4). SQ-535, when excited at $560 \mathrm{~nm}$, showed similar good PM staining properties (Figure S5) and thus constitutes a red shifted alternative to SP-468. KB cells stained with SP-468 and SQ-535 were then imaged under continuous laser scanning illumination for 10 minutes with negligible loss of intensity and with conserved PM staining thus demonstrating their photostability (movie 1 and 2). The PM staining efficiency of SP-468 was then assessed in fixed cells. We established that cell fixation followed by addition of the probe was preferable over the other way around and provided excellent results with no sign of internalization (Figure S6).

Additionally, the use of SQ-535 at only $500 \mathrm{nM}$ on fixed primary culture of hippocampal neurons and astrocytes in no wash conditions led to a homogeneous and bright staining of their PM (Figure 4A and B). 
bioRxiv preprint doi: https://doi.org/10.1101/819383. this version posted October 28,2019 . The copyright holder for this preprint (which was not certified by peer review) is the author/funder. It is made available under a CC-BY-NC-ND 4.0 International license.
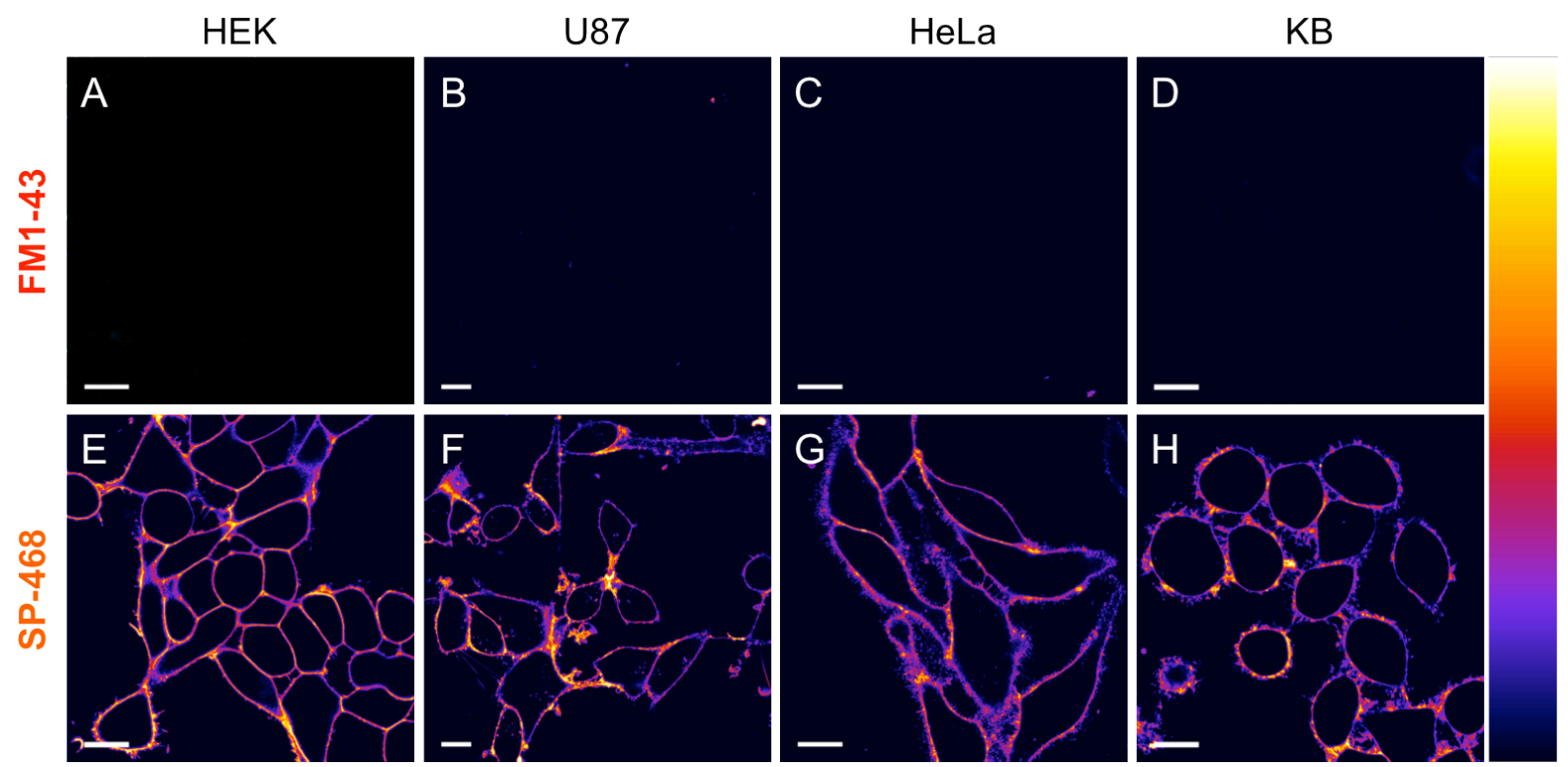

Figure 3. Laser scanning confocal imaging of live cells from four different cell lines stained with $1 \mu$ M of FM1-43 (A, B, C, D) and SP468 (E, F, G, H). Images were acquired 5 minutes after addition of the dyes in the medium. Excitation wavelength was $488 \mathrm{~nm}$ and the fluorescence signal was collected between 520 and $700 \mathrm{~nm}$. Scale bar is $15 \mu \mathrm{m}$. On the right is displayed the color lookup table.

The photostability and quality of staining allowed to obtaining high quality $3 \mathrm{D}$ images that revealed the dense neuronal network (Figure 4C). After incubation on fixed brain slices, SQ535 provided a homogeneous staining of the whole tissue (Figure 4D). When zoomed on the cortical layer, some dendritic processes were finely revealed in a multicolor $3 \mathrm{D}$ rendering (Figure 4D).

\section{Specificity and Cellular uptake}

We then aimed at comparing the specificity of the probes towards the PM. To this end, KB cells were co-stained with SQ$535(1 \mathrm{mM})$ and FM1-43 at a higher concentration $(5 \mathrm{mM})$ in order to increase its signal in imaging. After checking that crosstalk did not occur between the 2 channels in these conditions (Figure S7), a time laps imaging was performed for 10 minutes (Movie 3). The results first showed that FM1-43 at high concentration and after several minutes, provided a weak PM staining accompanied with non-specific staining of intracellular membranes (Figure 5A). Conversely, SQ-535 provided a fast and bright PM staining that kept its selectivity along the imaging period (Figure 5B, C and Movie 3).

In order to compare the fate of the probes over incubation, FM1-43 and SP-468 were incubated for $1 \mathrm{~h}$ at $37^{\circ} \mathrm{C}$ to provoke endocytosis. Imaging revealed that FM1-43 rapidly stained intracellular membranes along with endosomes vesicles (Figure S8A, movie 4). SP-468 kept a PM staining after incubation and showed punctiform staining in the cytoplasm with characteristic movement when imaged over the time (Movie 5) depicting endosomes (Figure S8B). To assess the difference in the internalization within the same cellular experiment, FM143 and SQ-535 were co-incubated in KB cells for $1 \mathrm{~h}$ at $37^{\circ} \mathrm{C}$ before being fixed. Analysis of images revealed that FM1-43 was actually distributed between endosomes and intracellular membranes (Figure 5D), whereas SQ-535 was distributed between the PM and endosomes (Figure 5E). Interestingly the merge images revealed that FM1-43 and SQ-535 do not fully follow the same internalization pathway as green and red signals poorly colocalized in the endosomes with a measured Pearson correlation coefficient of 0.32 (Figure 5F).
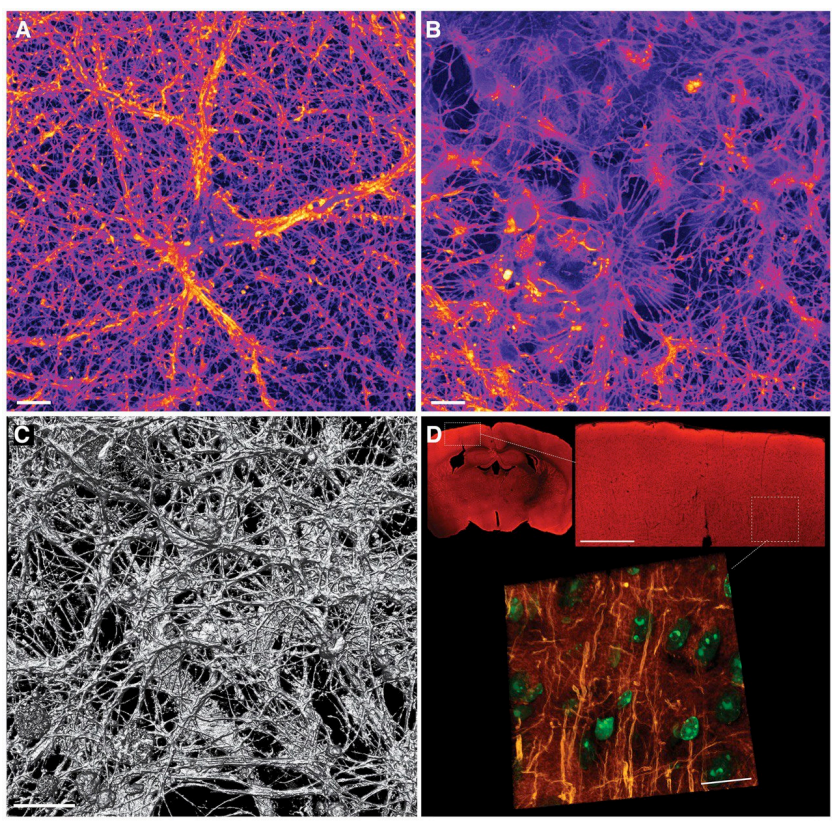

Figure 4. Laser scanning confocal imaging (with $20 \times$ objective) of fixed hippocampal neurons (A) and fixed astrocytic glial cells (B) incubated with SQ-535 $(500 \mathrm{nM})$. (C) 3D rendering of the complex neuronal network $(10 \mu \mathrm{m}$ thick $3 \mathrm{D}$ volume, $93 \times$ objective). (D) Fixed brain slice images using SQ-535 (in red) and DAPI (cell nuclei in green). Some dendritic processes within the cortical layer (top right inset, scale bar $500 \mu \mathrm{m}$ ) are revealed in the $3 \mathrm{D}$ rendering of a $12 \mu \mathrm{m}$ thick $\mathrm{Z}$ stack (scale bar: $20 \mu \mathrm{m}$ ). 


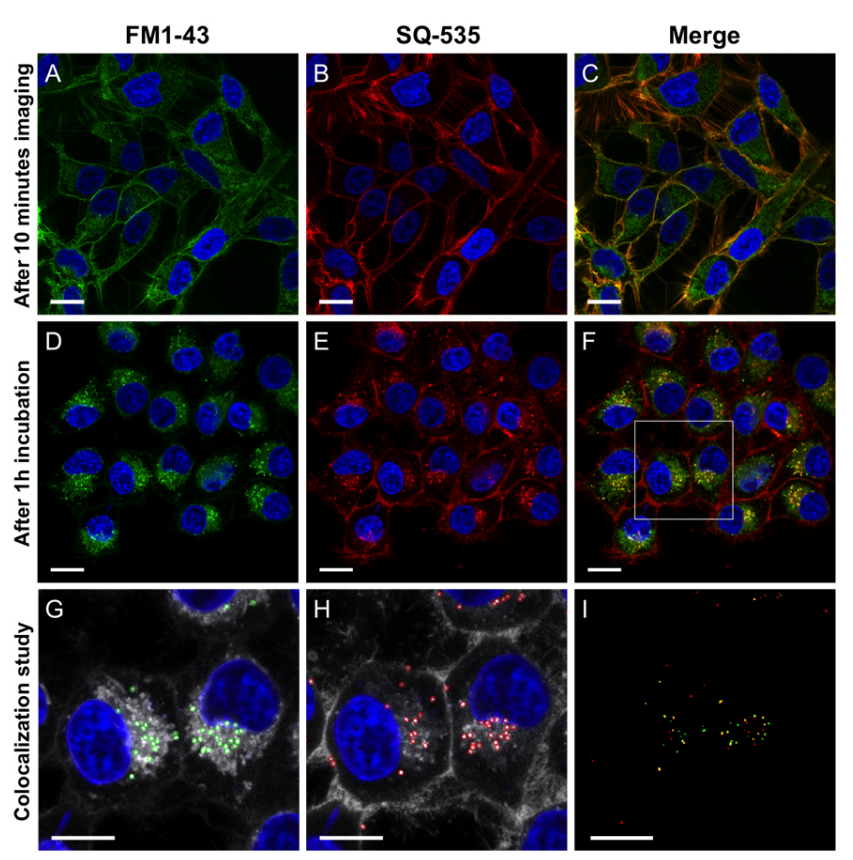

Figure 5. Laser scanning confocal imaging of $\mathrm{KB}$ cells stained with Hoechst $(5 \mu \mathrm{g} / \mathrm{mL})$ and $5 \mu \mathrm{M}$ of FM1-43 (A, D, G) and 1 $\mu \mathrm{M}$ SQ-535 (B, E, H). (A, B, C) Images of live cells after $10 \mathrm{~min}$ of continuous imaging. (D, E, F) Images of fixed cells after $1 \mathrm{~h}$ incubation in Opti-MEM. Merged channels (C, F) depict the different staining pattern and internalization pathway of the probes. $\mathrm{G}$ and $\mathrm{H}$ are zoomed images in the region of interest displayed in $\mathrm{F}$, highlighting endosomes using spots detection plugin. (I) The resulting merged binary images of the detected endosomes depicted a weak colocalization. Scale bars are $15 \mu \mathrm{m}$.

\section{PM staining of plant cells}

FM dyes are widely used in cellular plant imaging as it efficiently stains the PM of the latter and thus is useful for segmentation and for tracking trafficking vesicles. ${ }^{23,}{ }^{24} \mathrm{We}$ thus investigated on the ability of our modified FM probes to conserve efficient PM staining in plant cells. For this two different experiments were conducted. On one hand, a solution of probe was injected in the leaf of 6 weeks old Nicotiana Benthamiana plant (Figure 6A-D); on the other hand, whole seedlings were incubated in a solution of probe to stain the roots (Figure $6 \mathrm{E}$ F). The results showed that using the same imaging and processing conditions, FM1-43 and SP-468 both stained the PM and allowed segmentation of the cells thus showing that the newly developed PM probes can be used with both mammalian and plant cells. Noteworthy, although FM1-43 and SP-468 displayed similar staining properties in the roots, FM1-43 displayed slightly more homogeneous staining in leaf cells compared to SP-468 that displayed enhanced signal in stomata (Figure 6D).

\section{Conclusion}

Although FM dyes are known as plasma membrane probes, FM1-43 displayed rather weak PM staining properties in eukaryotic cells. We showed that by conserving the same core fluorophore and by means of rational chemical modifications using adapted targeting moieties and neutralizing the cationic nature of FM1-43, styryl dyes could be efficiently converted into PM probes with interesting properties for PM bioimaging. Notable improvements were obtained as the tendency for

crosstalk was reduced and the brightness as well as the photostability were significantly enhanced. The newly developed probes displayed fast, efficient and selective staining of PM in 5 different mammalian cell lines, including primary neurons, in contrast to parent FM1-43 that showed weak signal and significant intracellular staining. Remarkably, the new probes showed very good PM staining of fixed cells and brain slices. Additionally the ability to stain PM of plant cells was overall conserved making these new dyes adapted for both mammalian and plant cells experiments. The present work demonstrated the importance in the design of molecular fluorescent probes to fit with the bioimaging requirements. SP-468 and SQ-535 constitute reliable PM probes for users who want to benefit from large Stokes shift of PM markers with high photostability and to work with both live and fixed samples of cells and tissues.

FM1-43
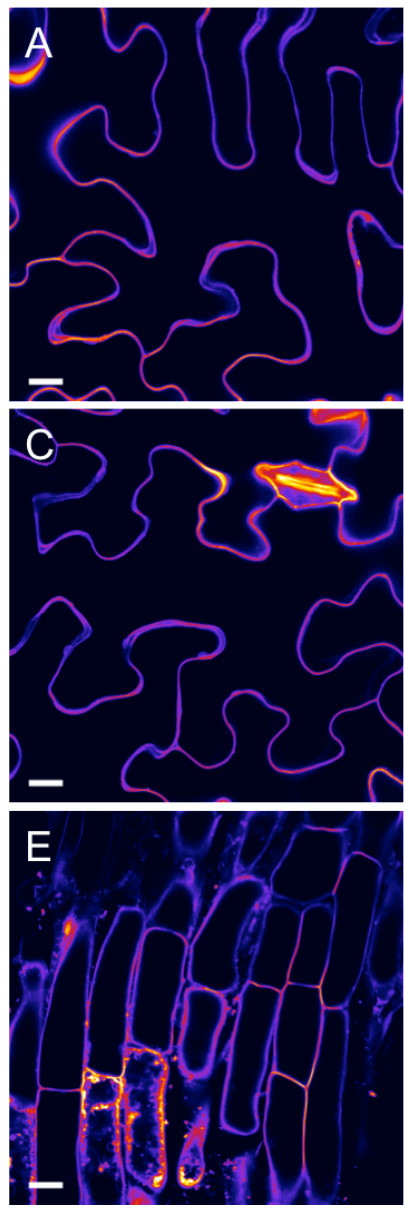

Figure 6. Laser scanning confocal imaging of plant cells (Nicotiana Benthamiana) stained with FM1-43 (A, C, E) and SP-468 (B, $\mathrm{D}, \mathrm{F})$. Images at the level of leafs without stomata (A, B) and with stomata $(\mathrm{C}, \mathrm{D})$. Images at the level of roots $(\mathrm{E}, \mathrm{F})$. Scale bar is 15 $\mu \mathrm{m}$. On the right is the color lookup table.

\section{Materials and methods}

Synthesis. All starting materials for synthesis were purchased from Alfa Aesar, Sigma Aldrich or TCI Europe and used as received unless stated otherwise. FM1-43 was purchased from Biotium. ${ }^{17}$ The solvents were not dried unless mentioned in 
the protocol. DOPC Liposomes of $\sim 100 \mathrm{~nm}$ were obtained following a described protocol after several rounds of extrusion. $^{33}$ NMR spectra were recorded on a Bruker Avance III $400 \mathrm{MHz}$ spectrometer. Mass spectra were obtained using an Agilent Q-TOF 6520 mass spectrometer. Synthesis of all new compounds is described in the supporting information.

Spectroscopy. For spectroscopy studies the concentration of the probes was $2 \mathrm{mM}$ and solvents were of spectroscopic grade. The water was miliQ water. Absorption and emission spectra were recorded on a Cary 400 Scan ultraviolet-visible spectrophotometer (Varian) and a FluoroMax-4 spectrofluorometer (Horiba Jobin Yvon) equipped with a thermostated cell compartment, respectively. For standard recording of fluorescence spectra, the emission was collected $10 \mathrm{~nm}$ after the excitation wavelength. All the spectra were corrected from wavelength-dependent response of the detector. The quantum yields were determined by comparison with fluorescein ${ }^{34}$ (in $\mathrm{NaOH} 0.1 \mathrm{M}, \mathrm{f}=0.95$ ) for FM1-43 and SP-468 (excitation at $460 \mathrm{~nm}$ ), and rhodamine $\mathrm{B}^{35}$ (in water, $\mathrm{f}=0.31$ ) for $\mathrm{SQ}-535$ (excitation at $520 \mathrm{~nm}$ ), following equation (1):

$$
\mathrm{QY}=\mathrm{QY} \mathrm{Y}_{R} \times \frac{I \times O D_{R} \times n^{2}}{I_{R} \times O D \times n_{R}^{2}}
$$

where QY is the quantum yield, I is the integrated fluorescence intensity, $\mathrm{n}$ is the refractive index, and OD is the optical density at the excitation wavelength. $\mathrm{R}$ represents the reference. The concentrations were adjusted by absorption considering the following extinction coefficients in $\mathrm{MeOH}$ : FM1-43, $52,000 \mathrm{M}^{-1} \cdot \mathrm{cm}^{-1}$ (according to the litterature ${ }^{36}$ ); SP-468, $50,000 \mathrm{M}^{-1} \cdot \mathrm{cm}^{-1}$ (determined from measured precursor SPdialkyne) and SQ-535, 49,800 $\mathrm{M}^{-1} \cdot \mathrm{cm}^{-1}$ (determined from measured precursor SQ-dialkyne).

Photostability assay. The dyes $(2 \mu \mathrm{M})$ in the presence of vesicles (100 eq of DOPC) in phosphate buffer ( $\mathrm{pH}$ 7.4) were all excited at the same optical density value based on their absorption spectra, namely @ $\mathrm{OD}=0.03$. FM1-43 was excited at $450 \mathrm{~nm}, \mathrm{SP}-468$ at $430 \mathrm{~nm}$ and SQ-535 at $478 \mathrm{~nm}$. Optical slits were set at $16 \mathrm{~mm}$ for excitation and $1 \mathrm{~mm}$ for emission. The emission was monitored at $600 \mathrm{~nm}$ for FM1-43 and SP468 and at 660 for SQ-535.

Membrane binding kinetics. A solution of DOPC $(200 \mu \mathrm{M})$ vesicles in $\mathrm{PB}$ at $\mathrm{pH} 7.4$ were stirred under excitation for 1 min, then $5 \mu \mathrm{L}$ of probe ( $5 \mu \mathrm{L}$ of stock at $400 \mu \mathrm{M}$ in DMSO) was added. Excitation was at $460 \mathrm{~nm}$ for FM 1-43 and SP-468 and the fluorescence was monitored at $600 \mathrm{~nm}$. For SQ-535 it was $520 \mathrm{~nm}$ and $660 \mathrm{~nm}$ respectively.

Cellular imaging. HEK293 (ATCC $®$ CRL-3216 ${ }^{\mathrm{TM}}$ ) and HeLa (ATCC ${ }^{\circledR}$ CCL-2 ${ }^{\mathrm{TM}}$ ) cells were grown in Dulbecco's Modified Eagle Medium without phenol red (DMEM, GibcoInvitrogen) supplemented with $10 \%$ fetal bovine serum (FBS, Lonza), 1\% L-Glutamine (Sigma Aldrich) and 1\% antibiotic solution (Penicillin-Streptomycin, Sigma-Aldrich) at $37^{\circ} \mathrm{C}$ in humidified atmosphere containing 5\% $\mathrm{CO}_{2}$. U87 (ATCC®) HTB-14 ${ }^{\mathrm{TM}}$ ) were grown in Minimum Essential Medium (MEM, Gibco-Invitrogen) supplemented with 10\% FBS, 1\% Ultra-Glutamine (Gibco-Invitrogen) and $1 \%$ antibiotic solution. KB cells (ATCC ${ }^{\circ}$ CCL-17) were grown DMEM (GibcoInvitrogen) supplemented with 10\% FBS (Lonza), 1\% non- essential amino acids (Gibco-Invitrogen), 1\% MEM vitamin solution (Gibco-Invitrogen), 1\% L-Glutamine (Sigma Aldrich) and $0.1 \%$ antibiotic solution (gentamicin, Sigma-Aldrich) at $37^{\circ} \mathrm{C}$ in humidified atmosphere containing $5 \% \mathrm{CO}_{2}$. Cells were seeded onto a chambered coverglass (IBiDi $\left.{ }^{\circledR}\right)$ at a density of $5 \times 10^{4}$ cells/well $24 \mathrm{~h}$ before the microscopy measurement. For imaging, the culture medium was removed and the attached cells were washed with Opti-MEM (GibcoInvitrogen). Next, the cells were incubated in Opti-MEM with Hoechst $(5 \mu \mathrm{g} / \mathrm{mL})$ to stain the nuclei for $1 \mathrm{~h} 00$. The cells were then washed with Opti-MEM and a freshly prepared solution of Styryl probe ( $2 \mathrm{mM}$ in Opti-MEM) was added to the cells prior to imaging. Confocal fluorescence images of the cells were taken on a Leica TSC SPE confocal microscope using a $63 \times$ oil immersion objective. The images were processed with Icy software. ${ }^{37}$ For colocalization between FM1-43 and SQ535 the following settings were used: FM1-43 (ex: $488 \mathrm{~nm}$, Em: 515-600 nm) and SQ-535 (ex: $561 \mathrm{~nm}$, Em: 650-750 nm). Colocalization study was performed with Icy software: Endosomes were localized by detecting intense spots using "spot detector" plugin. The obtained binary images of endosomes were merged and the "colocalization Studio" plugin ${ }^{38}$ was used to determine the colocalization Pearson's colocalization coefficient. Neuronal imaging: All animal experiments were performed in accordance with European Community guidelines legislation and reviewed by the local ethical committee of the Paris Diderot University. Hippocampal cultures from 18-day-old Sprague-Dawley rat embryos were prepared as described. ${ }^{39,40}$ Cells were dissociated by treatment with $0.25 \%$ trypsin for $15 \mathrm{~min}$ at $37^{\circ} \mathrm{C}$ and plated on poly-Ornithine (1 $\mathrm{mg} / \mathrm{mL}$, Sigma) coated glass coverslips in MEM supplemented with $10 \%$ horse serum, $0.6 \%$ glucose, $0.2 \%$ sodium bicarbonate, $2 \mathrm{mM}$ glutamine, and $10 \mathrm{IU} / \mathrm{mL}$ penicillin- streptomycin. After attachment neurons were grown in neurobasal medium supplemented with B27 (Thermo Fisher) conditioned on astroglia. Neurons were then imaged on Leica SP8 confocal microscope. For imaging, the medium was removed and the attached cells were washed with warm HBSS (GibcoInvitrogen) three times. Neurons were fixed with $4 \%$ PFA, $0.1 \%$ glutaraldehyde during $10 \mathrm{~min}$ at $37^{\circ} \mathrm{C}$. Samples were washed 3 times with HBSS and incubated with $500 \mathrm{nM} \mathrm{SQ}$ 535 and processed directly for confocal imaging in open chamber. Mice tissue: C57BL6/J mice were maintained on a $12 \mathrm{~h}$ light-dark cycle with ad libitum access to food and water. All animal work was conducted following protocol approved by ethical committee. Adult C57BL6 mice were euthanized and fixed with $4 \%$ paraformaldehyde by transcardial perfusion, post-fixed for $30 \mathrm{~min}$ in the same fixative, rinsed in PBS and kept in PBS- $30 \%$ sucrose at $4^{\circ} \mathrm{C}$ until use. Brains were sectioned on a cryotome at $40 \mathrm{~mm}$ and floating sections were processed for labeling as described for cultured neurons with slight modifications: slices were washed 3 times in PBS$\mathrm{NH}_{4} \mathrm{Cl} 50 \mathrm{mM}$ and incubated $3 \mathrm{~h}$ with DAPI $(10 \mathrm{mg} / \mathrm{mL})$ and SQ-535 $(8 \mu \mathrm{M})$ at room temperature under agitation. The microscope settings were: $405 \mathrm{~nm}$ laser for excitation of DAPI, emission was collected between 420 and $470 \mathrm{~nm}$. SQ-535 was excited with $561 \mathrm{~nm}$ laser line and fluorescence was collected with $650-750 \mathrm{~nm}$ detection range. The images were processed with LAsX and Icy software.

Cytotoxicity assay. Cytotoxicity assay was quantified by the MTT assay (3-(4,5-dimethylthiazol-2-yl)-2,5diphenyltetrazolium bromide). A total of $1 \times 10^{4} \mathrm{~KB}$ cells/well were seeded in a 96 -well plate $24 \mathrm{~h}$ prior to the cytotoxicity assay in Dulbecco's Modified Eagle Medium (Gibco Life 
Technologies -DMEM) complemented with $10 \%$ fetal bovine serum, Gentamicin $(100 \mu \mathrm{g} / \mathrm{mL})$, L-Glutamine $(2 \mathrm{mM})$, nonessential amino acids $(1 \mathrm{mM})$, MEM vitamin solution (1\%) and were incubated in a $5 \% \mathrm{CO}_{2}$ incubator at $37^{\circ} \mathrm{C}$. After medium removal, an amount of $100 \mu \mathrm{L}$ DMEM containing $5 \mu \mathrm{M}$, $1 \mu \mathrm{M}$ or $0.2 \mu \mathrm{M}$ of SMCy (SMCy3, SMCy3.5, SMCy5, SMCy5.5, SMCy7 and SMCy7.5) was added to the KB cell and incubated for $3 \mathrm{~h}$ at $37^{\circ} \mathrm{C}\left(5 \% \mathrm{CO}_{2}\right)$. As control, for each 96-well plate, the cells were incubated with DMEM containing the same percentage of DMSO $(0,5 \% \mathrm{v} / \mathrm{v})$ as the solution with the tested dyes or with Triton $1 \%$ as a positive control of cytotoxicity. After $24 \mathrm{~h}$ of dye incubation, the medium was replaced by $100 \mu \mathrm{L}$ of a mix containing DMEM + MTT solution (diluted in PBS beforehand) and the cells were incubated for $4 \mathrm{~h}$ at $37^{\circ} \mathrm{C}$. Then, $75 \mu \mathrm{L}$ of the mix was replaced by $50 \mu \mathrm{L}$ of DMSO (100\%) and gently shaken for $15 \mathrm{~min}$ at room temperature in order to dissolve the insoluble purple formazan reduced in living cells. The absorbance at $540 \mathrm{~nm}$ was measured (absorbances of the dyes at $540 \mathrm{~nm}$ were taken into account). Each concentration of dye was tested in sextuplicate in 3 independent assays. For each concentration, we calculated the percentage of cell viability in reference of the control DMEM+ $0.5 \%$ DMSO.

Plant imaging. Nicotiana Benthamiana were used as plant model and were obtained from the Institut de biologie moléculaire des plantes (IBMP) greenhouse. Staining of leaf cells: leaves from 5-6 weeks old plants were infiltrated with the help of a syringe containing a solution of probes ( $5 \mathrm{mM}$ in water). After 30 min, disk of leafs were taken off using a hole punch of $0.7 \mathrm{~cm}$ in diameter. The leaf samples were maintained in water between cover slips and were directly imaged. Staining of root cells: young plants ( 3 to 5 days after germination) were placed in $1 \mathrm{~mL}$ solution of probe (5 $\mathrm{mM}$ in water) for 1 hour at RT. The roots were washed with water and placed under cover slips before being imaged.

\section{Acknowledgements}

This work was supported by the European Research Council ERC Consolidator grant BrightSens 648528 and ANR BrightRiboProbes (ANR-16-CE11-0010). The authors would like to thank Neurimag (Paris) and Romain Vauchelles for his assistance at the PIQ platform as well as the PACSI analysis platform. We are grateful to Leducq foundation for its support concerning NeurImag SP8 confocal system purchase. We are also grateful to IBMP's gardener and Elodie Klein for providing the plants and Dmytro Danylchuk for the preparation of liposomes.

\section{ASSOCIATED CONTENT}

Data on synthesis protocol, ${ }^{1} \mathrm{H}$ and ${ }^{13} \mathrm{C}$ NMR and mass spectra, spectroscopy, additional cellular imaging, and cytotoxicity assays. Movie 1. Photostability SP-468 (AVI)

Movie 2. Photostability SQ-535 (AVI)

Movie 3. FM1-43 \& SQ-535 time laps (AVI)

Movie 4. Incubation of FM-1-43 (AVI)

Movie 5. Incubation of SP-468 (AVI)

\section{Conflict of interest}

The authors declare no conflict of interest.

\section{References}

(1) Lingwood, D.; Simons, K. Lipid Rafts As a MembraneOrganizing Principle. Science 2010, 327 (5961), 46-50. https://doi.org/10.1126/science.1174621.

(2) Sezgin, E.; Levental, I.; Mayor, S.; Eggeling, C. The Mystery of Membrane Organization: Composition, Regulation and Roles of Lipid Rafts. Nat. Rev. Mol. Cell Biol. 2017, 18 (6), 361-374. https://doi.org/10.1038/nrm.2017.16.

(3) Rello, S.; Stockert, J. C.; Moreno, V.; Gámez, A.; Pacheco, M.; Juarranz, A.; Cañete, M.; Villanueva, A. Morphological Criteria to Distinguish Cell Death Induced by Apoptotic and Necrotic Treatments. Apoptosis 2005, $10 \quad$ (1), 201-208. https://doi.org/10.1007/s10495-005-6075-6.

(4) Revelo, N. H.; Kamin, D.; Truckenbrodt, S.; Wong, A. B.; Reuter-Jessen, K.; Reisinger, E.; Moser, T.; Rizzoli, S. O. A New Probe for Super-Resolution Imaging of Membranes Elucidates Trafficking Pathways. J. Cell Biol. 2014, 205 (4), 591-606. https://doi.org/10.1083/jcb.201402066.

(5) Kwiatek, J. M.; Owen, D. M.; Abu-Siniyeh, A.; Yan, P.; Loew, L. M.; Gaus, K. Characterization of a New Series of Fluorescent Probes for Imaging Membrane Order. Plos One 2013, 8 (2), e52960. https://doi.org/10.1371/journal.pone.0052960.

(6) Zhang, X.; Wang, C.; Jin, L.; Han, Z.; Xiao, Y. Photostable Bipolar Fluorescent Probe for Video Tracking Plasma Membranes Related Cellular Processes. ACS Appl. Mater. Interfaces 2014, 6 (15), 12372-12379. https://doi.org/10.1021/am503849c.

(7) Heek, T.; Nikolaus, J.; Schwarzer, R.; Fasting, C.; Welker, P.; Licha, K.; Herrmann, A.; Haag, R. An Amphiphilic Perylene Imido Diester for Selective Cellular Imaging. Bioconjug. Chem. 2013, 24 (2), 153-158. https://doi.org/10.1021/bc3005655.

(8) Collot, M.; Ashokkumar, P.; Anton, H.; Boutant, E.; Faklaris, O.; Galli, T.; Mély, Y.; Danglot, L.; Klymchenko, A. S. MemBright: A Family of Fluorescent Membrane Probes for Advanced Cellular Imaging and Neuroscience. Cell Chem. Biol. 0 (0). https://doi.org/10.1016/j.chembiol.2019.01.009.

(9) Collot, M.; Boutant, E.; Lehmann, M.; Klymchenko, A. S. BODIPY with Tuned Amphiphilicity as a Fluorogenic Plasma Membrane Probe. Bioconjug. Chem. 2019, 30 (1), 192-199. https://doi.org/10.1021/acs.bioconjchem.8b00828.

(10) Collot, M.; Kreder, R.; Tatarets, A. L.; Patsenker, L. D.; Mely, Y.; Klymchenko, A. S. Bright Fluorogenic Squaraines with Tuned Cell Entry for Selective Imaging of Plasma Membrane vs. Endoplasmic Reticulum. Chem. Commun. 2015, 51 (96), 1713617139. https://doi.org/10.1039/C5CC06094J.

(11) Shaya, J.; Collot, M.; Bénailly, F.; Mahmoud, N.; Mély, Y.; Michel, B. Y.; Klymchenko, A. S.; Burger, A. Turn-on Fluorene Push-Pull Probes with High Brightness and Photostability for Visualizing Lipid Order in Biomembranes. ACS Chem. Biol. 2017, 12 (12), 3022-3030. https://doi.org/10.1021/acschembio.7b00658.

(12) Kucherak, O. A.; Oncul, S.; Darwich, Z.; Yushchenko, D. A.; Arntz, Y.; Didier, P.; Mély, Y.; Klymchenko, A. S. Switchable Nile Red-Based Probe for Cholesterol and Lipid Order at the Outer Leaflet of Biomembranes. J. Am. Chem. Soc. 2010, 132 (13), 49074916. https://doi.org/10.1021/ja100351w.

(13) Klymchenko, A. S.; Kreder, R. Fluorescent Probes for Lipid Rafts: From Model Membranes to Living Cells. Chem. Biol. 2014, 21 (1), 97-113. https://doi.org/10.1016/j.chembiol.2013.11.009.

(14) Collot, M.; Ashokkumar, P.; Anton, H.; Boutant, E.; Faklaris, O.; Galli, T.; Mély, Y.; Danglot, L.; Klymchenko, A. S. MemBright: A Family of Fluorescent Membrane Probes for Advanced Cellular Imaging and Neuroscience. Cell Chem. Biol. 2019, 26 (4), 600-614.e7. https://doi.org/10.1016/j.chembiol.2019.01.009.

(15) Danylchuk, D. I.; Moon, S.; Xu, K.; Klymchenko, A. S. Switchable Solvatochromic Probes for Live-Cell Super-Resolution Imaging of Plasma Membrane Organization. Angew. Chem. Int. Ed. 2019, 58 (42), 14920-14924. https://doi.org/10.1002/anie.201907690.

(16) Betz, W. J.; Mao, F.; Bewick, G. S. Activity-Dependent Fluorescent Staining and Destaining of Living Vertebrate Motor Nerve Terminals. J. Neurosci. 1992, 12 (2), 363-375. https://doi.org/10.1523/JNEUROSCI.12-02-00363.1992.

(17) Life Technologies https://www.thermofisher.com/.

(18) Biotium Https://Biotium.Com/. 
bioRxiv preprint doi: https://doi.org/10.1101/819383. this version posted October 28,2019 . The copyright holder for this preprint (which was not certified by peer review) is the author/funder. It is made available under a CC-BY-NC-ND 4.0 International license.

(19) Wuskell, J. P.; Boudreau, D.; Wei, M.; Jin, L.; Engl, R.; Chebolu, R.; Bullen, A.; Hoffacker, K. D.; Kerimo, J.; Cohen, L. B.; et al. Synthesis, Spectra, Delivery and Potentiometric Responses of New Styryl Dyes with Extended Spectral Ranges. J. Neurosci. Methods 2006, $\quad 151 \quad$ (2), $200-215$. https://doi.org/10.1016/j.jneumeth.2005.07.013.

(20) Yan, P.; Acker, C. D.; Zhou, W.-L.; Lee, P.; Bollensdorff, C.; Negrean, A.; Lotti, J.; Sacconi, L.; Antic, S. D.; Kohl, P.; et al. Palette of Fluorinated Voltage-Sensitive Hemicyanine Dyes. Proc. Natl. Acad. Sci. 2012, 109 (50), 20443-20448. https://doi.org/10.1073/pnas.1214850109.

(21) Jin, L.; Millard, A. C.; Wuskell, J. P.; Dong, X.; Wu, D.; Clark, H. A.; Loew, L. M. Characterization and Application of a New Optical Probe for Membrane Lipid Domains. Biophys. J. 2006, 90 (7), 2563. https://doi.org/10.1529/biophysj.105.072884.

(22) Schote, U.; Seelig, J. Interaction of the Neuronal Marker Dye FM1-43 with Lipid Membranes: Thermodynamics and Lipid Ordering. Biochim. Biophys. Acta BBA - Biomembr. 1998, 1415 (1), 135-146. https://doi.org/10.1016/S0005-2736(98)00188-6.

(23) Bolte, S.; Talbot, C.; Boutte, Y.; Catrice, O.; Read, N. D.; Satiat-Jeunemaitre, B. FM-Dyes as Experimental Probes for Dissecting Vesicle Trafficking in Living Plant Cells. J. Microsc. 2004, 214 (2), 159-173. https://doi.org/10.1111/j.0022-2720.2004.01348.x.

(24) Jelínková, A.; Malínská, K.; Simon, S.; Kleine-Vehn, J.; Pařezová, M.; Pejchar, P.; Kubeš, M.; Martinec, J.; Friml, J.; Zažímalová, E.; et al. Probing Plant Membranes with FM Dyes: Tracking, Dragging or Blocking? Plant J. 2010, 61 (5), 883-892. https://doi.org/10.1111/j.1365-313X.2009.04102.x.

(25) Jennings, P.; Bertocchi, C.; Frick, M.; Haller, T.; Pfaller, W.; Dietl, P. Ca2+ Induced Surfactant Secretion in Alveolar Type II Cultures Isolated from the H-2Kb-TsA58 Transgenic Mouse. Cell. Physiol. Biochem. Int. J. Exp. Cell. Physiol. Biochem. Pharmacol. 2005, 15 (1-4), 159-166. https://doi.org/10.1159/000083648.

(26) Wiederkehr, A.; Avaro, S.; Prescianotto-Baschong, C.; Haguenauer-Tsapis, R.; Riezman, H. The F-Box Protein Rcylp Is Involved in Endocytic Membrane Traffic and Recycling out of an Early Endosome in Saccharomyces Cerevisiae. J. Cell Biol. 2000, 149 (2), 397-410. https://doi.org/10.1083/jcb.149.2.397.

(27) Murthy, V. N.; Stevens, C. F. Synaptic Vesicles Retain Their Identity through the Endocytic Cycle. Nature 1998, 392 (6675), 497-501. https://doi.org/10.1038/33152.

(28) Betz, W. J.; Bewick, G. S. Optical Analysis of Synaptic Vesicle Recycling at the Frog Neuromuscular Junction. Science 1992, 255 (5041), 200-203. https://doi.org/10.1126/science.1553547.

(29) Rea, R.; Li, J.; Dharia, A.; Levitan, E. S.; Sterling, P.; Kramer, R. H. Streamlined Synaptic Vesicle Cycle in Cone Photoreceptor Terminals. Neuron 2004, 41 (5), 755-766. https://doi.org/10.1016/s0896-6273(04)00088-1.

(30) Darwich, Z.; Klymchenko, A. S.; Dujardin, D.; Mély, Y. Imaging Lipid Order Changes in Endosome Membranes of Live Cells by Using a Nile Red-Based Membrane Probe. RSC $A d v$. 2014, 4 (17), 8481-8488. https://doi.org/10.1039/C3RA47181K.

(31) Sednev, M. V.; Belov, V. N.; Hell, S. W. Fluorescent Dyes with Large Stokes Shifts for Super-Resolution Optical Microscopy of Biological Objects: A Review. Methods Appl. Fluoresc. 2015, 3 (4), 042004. https://doi.org/10.1088/2050-6120/3/4/042004.

(32) Sezgin, E.; Schneider, F.; Zilles, V.; Urbančič, I.; Garcia, E.; Waithe, D.; Klymchenko, A. S.; Eggeling, C. Polarity-Sensitive Probes for Superresolution Stimulated Emission Depletion Microscopy. Biophys. J. 2017, 113 (6), 1321-1330 https://doi.org/10.1016/j.bpj.2017.06.050.

(33) Lin, J.; Alexander-Katz, A. Cell Membranes Open "Doors" for Cationic Nanoparticles/Biomolecules: Insights into Uptake Kinetics. ACS Nano 2013, 7 (12), 10799-10808. https://doi.org/10.1021/nn4040553.

(34) Jędrzejewska, B.; Pietrzak, M.; Pączkowski, J. Solvent Effects on the Spectroscopic Properties of Styrylquinolinium Dyes Series. J. Fluoresc. 2010, 20 (1), 73-86. https://doi.org/10.1007/s10895009-0524-5.

(35) Hope, M. J.; Bally, M. B.; Webb, G.; Cullis, P. R. Production of Large Unilamellar Vesicles by a Rapid Extrusion Procedure: Characterization of Size Distribution, Trapped Volume and Ability to Maintain a Membrane Potential. Biochim. Biophys. Acta 1985, 812 (1), 55-65.

(36) Lakowicz, J. R. Principles of Fluorescence Spectroscopy; Springer Science \& Business Media, 2007.

(37) Magde, D.; Rojas, G. E.; Seybold, P. G. Solvent Dependence of the Fluorescence Lifetimes of Xanthene Dyes. Photochem. Photobiol. 1999, 70 (5), 737-744. https://doi.org/10.1111/j.17511097.1999.tb08277.x.

(38) Schote, U.; Seelig, J. Interaction of the Neuronal Marker Dye FM1-43 with Lipid Membranes: Thermodynamics and Lipid Ordering. Biochim. Biophys. Acta BBA - Biomembr. 1998, 1415 (1), 135-146. https://doi.org/10.1016/S0005-2736(98)00188-6.

(39) Chaumont, F. de; Dallongeville, S.; Chenouard, N.; Hervé, N.; Pop, S.; Provoost, T.; Meas-Yedid, V.; Pankajakshan, P.; Lecomte, T.; Montagner, Y. L.; et al. Icy: An Open Bioimage Informatics Platform for Extended Reproducible Research. Nat. Methods 2012, 9 (7), nmeth.2075. https://doi.org/10.1038/nmeth.2075.

(40) Lagache, T.; Sauvonnet, N.; Danglot, L.; Olivo-Marin, J.C. Statistical Analysis of Molecule Colocalization in Bioimaging. Cytom. Part J. Int. Soc. Anal. Cytol. 2015, 87 (6), 568-579. https://doi.org/10.1002/cyto.a.22629.

(41) Banker, G. A.; Cowan, W. M. Rat Hippocampal Neurons in Dispersed Cell Culture. Brain Res. 1977, 126 (3), 397-342. https://doi.org/10.1016/0006-8993(77)90594-7.

(42) Danglot, L.; Triller, A.; Bessis, A. Association of Gephyrin with Synaptic and Extrasynaptic GABAA Receptors Varies during Development in Cultured Hippocampal Neurons. Mol. Cell. Neurosci. 2003, 23 (2), 264-278. 
bioRxiv preprint doi: https://doi.org/10.1101/819383. this version posted October 28, 2019. The copyright holder for this preprint (which was not certified by peer review) is the author/funder. It is made available under a CC-BY-NC-ND 4.0 International license.

Table of content graphic (TOC)

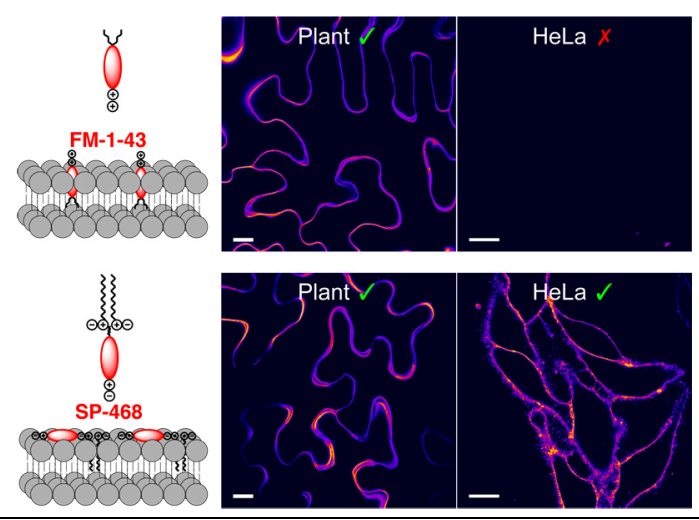

